\title{
A histological evaluation and in vivo assessment of intratumoral near infrared photothermal nanotherapy-induced tumor regression
}

This article was published in the following Dove Press journal:

International Journal of Nanomedicine

5 Novermber 2014

Number of times this article has been viewed

\author{
Hadiyah N Green ${ }^{1,2}$ \\ Stephanie D Crockett ${ }^{3}$ \\ Dmitry V Martyshkin' \\ Karan P Singh ${ }^{2,4}$ \\ William E Grizzle ${ }^{2,5}$ \\ Eben L Rosenthal ${ }^{2,6}$ \\ Sergey B Mirov'
}

'Department of Physics, Center for Optical Sensors and Spectroscopies, ${ }^{2}$ Comprehensive Cancer Center, ${ }^{3}$ Department of Pediatrics, Division of Neonatology, ${ }^{4}$ Department of Medicine, Division of Preventive Medicine, Biostatistics and Bioinformatics Shared Facility, ${ }^{5}$ Department of Pathology, ${ }^{6}$ Department of Surgery, Division of Otolaryngology, Head and Neck Surgery, The University of Alabama at Birmingham, Birmingham, AL, USA
Correspondence: Hadiyah N Green Department of Materials Science and Engineering, Tuskegee University 1200 W Montgomery Road,

Tuskegee, AL 36088, USA

$\mathrm{Tel}+\mathrm{I} 3347244307$

Fax +I 3347244424

Email hgreen@mytu.tuskegee.edu
Purpose: Nanoparticle (NP)-enabled near infrared (NIR) photothermal therapy has realized limited success in in vivo studies as a potential localized cancer therapy. This is primarily due to a lack of successful methods that can prevent NP uptake by the reticuloendothelial system, especially the liver and kidney, and deliver sufficient quantities of intravenously injected NPs to the tumor site. Histological evaluation of photothermal therapy-induced tumor regression is also neglected in the current literature. This report demonstrates and histologically evaluates the in vivo potential of NIR photothermal therapy by circumventing the challenges of intravenous NP delivery and tumor targeting found in other photothermal therapy studies.

Methods: Subcutaneous Cal 27 squamous cell carcinoma xenografts received photothermal nanotherapy treatments, radial injections of polyethylene glycol (PEG)-ylated gold nanorods and one NIR $785 \mathrm{~nm}$ laser irradiation for 10 minutes at $9.5 \mathrm{~W} / \mathrm{cm}^{2}$. Tumor response was measured for 10-15 days, gross changes in tumor size were evaluated, and the remaining tumors or scar tissues were excised and histologically analyzed.

Results: The single treatment of intratumoral nanorod injections followed by a 10 minute NIR laser treatment also known as photothermal nanotherapy, resulted in $\sim 100 \%$ tumor regression in $\sim 90 \%$ of treated tumors, which was statistically significant in a comparison to the average of all three control groups over time $(P<0.01)$.

Conclusion: Photothermal nanotherapy, or intratumoral nanorod injections followed by NIR laser irradiation of tumors and tumor margins, demonstrate the potential of NIR photothermal therapy as a viable localized treatment approach for primary and early stage tumors, and prevents NP uptake by the reticuloendothelial system.

Keywords: photothermal cancer therapy, malignancy, cancer treatment, intratumoral, gold nanorods, nanoparticles, PEGylation, laser therapy

\section{Introduction}

New approaches are needed to selectively eradicate malignancies and provide local, alternative, or supplemental treatments to the current standards of cancer therapy. A variety of local, intratumoral therapies have been developed to provide advantages over surgery and chemo-radiation, that include minimal invasiveness, minimal side effects, and shorter hospitalizations. ${ }^{1-4}$ These therapies are based on different physical mechanisms and ablation techniques including microwave, ${ }^{5-7}$ focused ultrasound, ${ }^{8-10}$ radiofrequency, ${ }^{11,12}$ and cryoablation ${ }^{13-15}$ therapies. The disadvantages of these therapies include limited specificity, limited delivery, and tumor recurrence. ${ }^{1-4}$ 
An emerging local therapy that could address the disadvantages of other intratumoral therapies is near infrared (NIR) photothermal therapy (PTT). ${ }^{16-18}$ NIR PTT is a minimally invasive local therapy that is distinguished from other local therapies because it utilizes gold nanoparticles (AuNPs), such as nanospheres, ${ }^{19-21}$ nanocages, ${ }^{22,23}$ and nanorods, ${ }^{24-26}$ as thermal-contrast agents to provide sitespecific thermal responses in combination with otherwise harmless laser irradiation. ${ }^{27-32}$ The NIR laser emits light in a spectral range that is transparent to normal tissue and overlaps with the plasmon resonance absorption of the AuNPs. ${ }^{27,33}$ AuNPs absorb NIR light energy and convert rapid electron oscillations into thermal energy, resulting in a therapeutic increase in temperature to $>40^{\circ} \mathrm{C}$, and consequent death of tumor cells. ${ }^{27,34}$ NIR PTT has an advantage over other local therapies because it provides compounding specificity to the tumor and its margin, and a controlled depth of penetration. ${ }^{35,36}$

The therapeutic properties of PTT have been clearly demonstrated in vitro. ${ }^{19,37,38}$ However, the success PTT has realized in vitro has yet to be achieved in vivo, because many prior approaches have relied on the passive delivery of untargeted nanoparticles (NPs) to the tumor. ${ }^{20,25,35}$ These approaches rely on the enhanced permeability and retention effect, ${ }^{39-41}$ a characteristic of tumor vasculature, which allows passive delivery of NPs to the site of the tumor and enables NP accumulation in the tumor due to the leaky vasculature of the tumor. Recently, NP delivery strategies that incorporate NP conjugation to a tumor-targeting ligand have been implemented in efforts to improve NP delivery. ${ }^{42,43}$ Regardless of the NP delivery mechanisms used, uptake by the reticuloendothelial system (RES), particularly the liver, spleen, and kidney, prevent up to $90 \%$ of systemically injected NPs from successfully reaching the tumor. ${ }^{44-49}$ New approaches in NP conjugation technology are needed to optimize NP delivery to the tumor. Further, to our knowledge, many studies using NP-enabled NIR photothermal therapy have not included a rigorous histological analysis of treated tumors, thus creating a knowledge gap of the effect of photothermal therapy at the cellular level. ${ }^{16-26}$ This study demonstrates the potential therapeutic efficacy of NIR photothermal therapy by circumventing the inefficiencies encountered with systemic NP delivery methods. We report the tumor regression resulting from photothermal nanotherapy (PNT), or intratumorally injected NPs followed by 10 minutes of NIR laser irradiation, evaluated by statistical analysis and characterized histologically.

\section{Materials and methods Gold nanorod fabrication}

Gold nanorods (AuNRs) were fabricated according to the method described by Sau and Murphy ${ }^{50}$ and modified by Green et al. ${ }^{51}$ Stock solutions were made by first cooling $30 \mathrm{~mL}$ of high performance liquid chromatography grade water to $0^{\circ} \mathrm{C}$. Solutions of $1 \mathrm{mM}$ gold (III) chloride trihydrate, $\left(\mathrm{HAuCl}_{4} \cdot 3 \mathrm{H}_{2} \mathrm{O}\right)$ (Sigma-Aldrich, St Louis, MO, USA), $200 \mathrm{mM}$ cetyltrimethylammonium bromide (CTAB) (Sigma-Aldrich), $78.8 \mathrm{mM}$ ascorbic acid, and $32 \mathrm{mM} \mathrm{AgNO}_{3}$ (Sigma-Aldrich) were then prepared. A hot-water bath, $\sim 50^{\circ} \mathrm{C}$, was used to dissolve CTAB in an aqueous solution. Ice-cold distilled water was rapidly added to $10 \mathrm{mM} \mathrm{NaBH}_{4}$ and the solution was returned to ice. Seeding solution was made by combining $2.5 \mathrm{~mL}$ of $1 \mathrm{mM} \mathrm{HAuCl} \cdot 3 \mathrm{H}_{2} \mathrm{O}, 5 \mathrm{~mL}$ of $200 \mathrm{mM}$ $\mathrm{CTAB}$, and $0.6 \mathrm{~mL}$ of ice-cold $10 \mathrm{mM} \mathrm{NaBH}_{4}$, and the resulting solution was incubated at room temperature $\left(25^{\circ} \mathrm{C}\right)$ for 2 hours before use. Growth solution was prepared by mixing $20 \mathrm{~mL}$ of $1 \mathrm{mM} \mathrm{HAuCl}_{4} \cdot 3 \mathrm{H}_{2} \mathrm{O}$ and $20 \mathrm{~mL}$ of $200 \mathrm{mM} \mathrm{CTAB}$ with $32 \mathrm{mM} \mathrm{AgNO}_{3}, 78.8 \mathrm{mM}$ ascorbic acid, and seeding solution. Once the color of the solution changed to a deep pink color, the nanorods were left to grow undisturbed for 2 hours at room temperature $\left(25^{\circ} \mathrm{C}\right)$.

\section{Gold nanorod biofunctionalization using polyethylene glycol}

AuNRs were fabricated as described in the "Gold nanorod fabrication" section with the surfactant, CTAB, as a capping agent to control the size of the nanorod. To increase in vivo nanorod biocompatibility and stability, polyethylene glycol (PEG) was used to replace CTAB on the surface of nanorods in a process known as "PEGylation". A covalent bond forms between the thiol group of PEG and the surface of the AuNR, replacing CTAB. PEGylation is advantageous because it decreases immunogenicity and adsorption to the negatively charged luminal surface of blood vessels and suppresses non-specific binding to charged molecules. ${ }^{52,53}$ During the CTAB removal process, nanorods were centrifuged at 7,000 $\mathrm{g}$ for 20 minutes, decanted, and the resulting pellet was resuspended in $100 \mathrm{mM}$ phosphate buffered saline. The nanorods were then PEGylated using $1 \mathrm{mM}$ of thiol-terminated methoxy-PEG (molecular weight $=5,000$ : Nanocs, New York, NY, USA) and $2 \mathrm{mM}$ of potassium carbonate (Gibco; Life Technologies, Carlsbad, CA, USA). The mixture was then incubated overnight. ${ }^{51,53}$ The maximum peak of plasmon resonance absorption for different batches of AuNRs averaged at $\lambda=784 \mathrm{~nm}$ as measured by 
an ultra violet-visible-NIR spectrophotometer (UV 3101PC; Shimadzu Corporation, Kyoto, Japan) (Figure 1A). A transmission electron microscope (TEM) (Tecnai ${ }^{\mathrm{TM}} \mathrm{T} 12$, $80 \mathrm{kV}$ or $120 \mathrm{kV}$; FEI, Hillsboro, OR, USA) was used to examine the AuNRs' morphology, size, and consistency

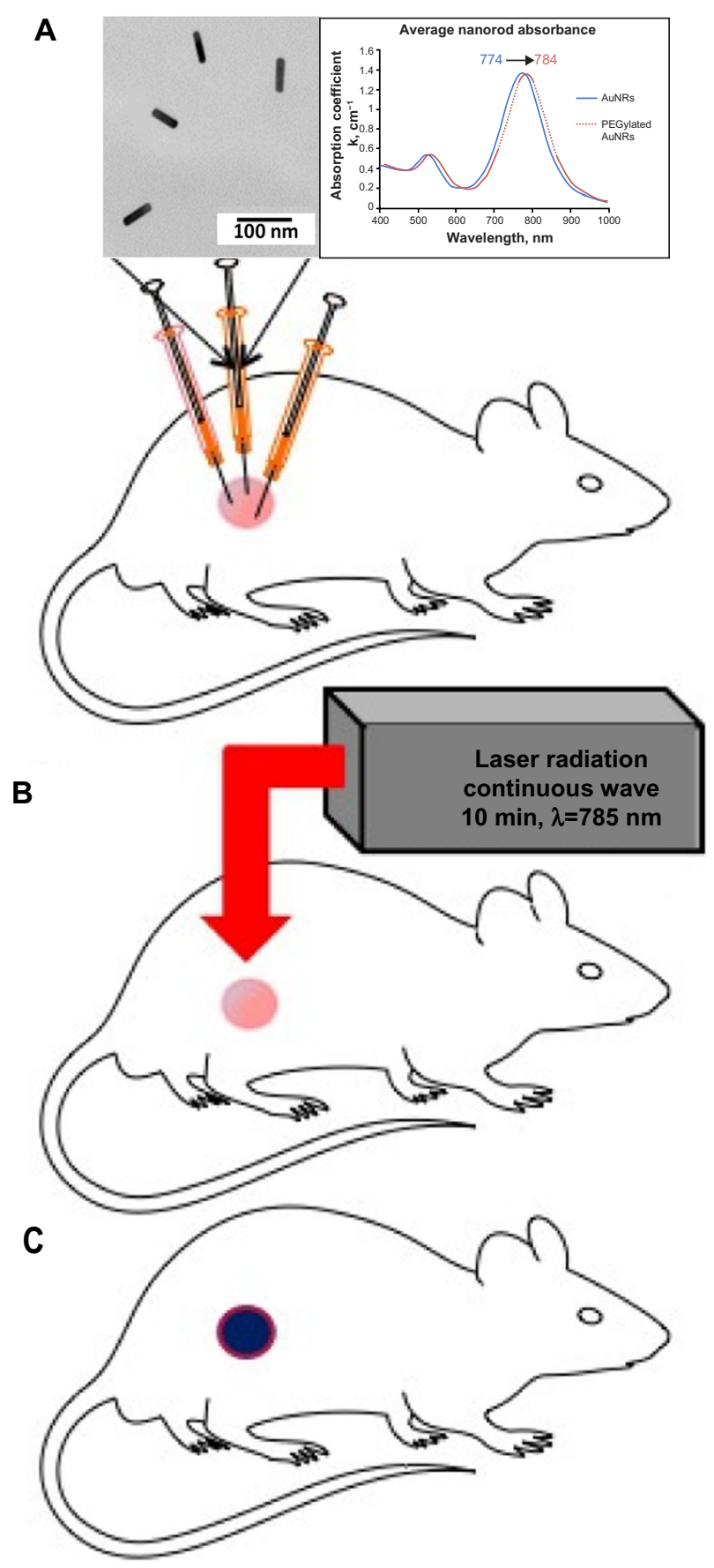

Figure I Schematic representation of treatment platform.

Notes: (A) PEGylated AuNRs, transmission electron microscope images, and $785 \mathrm{~nm}$ peak absorption spectra shown, were used in multiple radial intratumoral injections, followed by (B), a single 10 minute near infrared laser irradiation of tumor resulting in (C), tumor discoloration and eventual tumor regression. Abbreviations: PEG, polyethylene glycol; AuNRs, gold nanorods. using carbon-only copper grids (Electron Microscopy Sciences, Hatfield, PA, USA) and uranyl acetate stain (Polysciences, Inc., Warrington, PA, USA). Digital images of AuNRs were captured on a $2 \mathrm{k}$ resolution (2,000 pixels) camera (Advanced Microscopy Techniques, Corp, Woburn, MA, USA) and averaged $\sim 40 \mathrm{~nm} \times 10 \mathrm{~nm}$ in size, with an aspect ratio of $\sim 4$ (Figure 1).

\section{Tumor treatments}

Cal 27, an oral, head, and neck squamous cell carcinoma line, was maintained in Dulbecco's Modified Eagle's Medium containing $10 \%$ fetal bovine serum, supplemented with L-glutamine, penicillin, and streptomycin, and incubated at $37^{\circ} \mathrm{C}$ in $5 \% \mathrm{CO}_{2}$. Approximately $1 \times 10^{6} \mathrm{Cal} 27$ cells $/ 100 \mu \mathrm{L}$ of serum-free media were injected subcutaneously into athymic nude female mice for in vivo experiments and allowed to grow until the tumors reached $\sim 5 \mathrm{~mm}$ in diameter.

Photothermal nanotherapy was performed according to the treatment approach illustrated in Figure 1. Each tumor received intratumoral injections radially at multiple sites with various amounts of PEGylated nanorod solution, $\sim 10-50 \mu \mathrm{L}$, until the tumor reached maximum injection capacity, further injection would have compromised the integrity of the tumor. There were no observable differences in tumor regression for different volumes of AuNRs used, thus no dose curve was observed. Tumors were laser irradiated immediately (within 30 minutes) after AuNR injections, if they were in the group designated to receive the combination of nanorods and laser irradiation treatment. A continuous wave NIR semiconductor diode laser of $785 \mathrm{~nm}$ wavelength and $9.5 \mathrm{~W} / \mathrm{cm}^{2}$ fluence (SDL, Inc., San Jose, CA, USA) was used to irradiate the tumors for a single exposure time of 10 minutes. The beam diameter was adjusted to include the diameter of the tumor. Equivalent parameters were used in all in vitro studies, except when cells adherent to plate wells were irradiated for 4 minutes and analyzed using a trypan blue live/dead assay (Fischer Scientific, Fair Lawn, NJ, USA) and a flow cytometer (BD, Franklin Lakes, NJ, USA).

\section{Gross anatomy analysis}

Tumor responses were measured with digital calipers and captured with photography. Changes in tumor volume were calculated based on the percentage of increase or decrease from the tumor volume at the start of the treatment, using this initial tumor volume as a $100 \%$ value. At the end of the study, mice were euthanized, and tumors or remaining scars were excised to evaluate the combined effect of AuNRs and the NIR laser in comparison to the three 
control groups: no-treatment; laser-only; and AuNR-only options.

\section{Histological analysis}

After the treatments, any remaining tumor or the areas where the tumors were located, and the surrounding nontumor tissues were completely excised and fixed in $10 \%$ neutral buffered formalin at room temperature $\left(25^{\circ} \mathrm{C}\right)$ overnight. The tumor tissue sections were processed and embedded in paraffin blocks. Five-micron sections of the formalin-fixed, paraffin-embedded tissue blocks were cut with a microtome (Accu-Cut SRM 200; Sakura Finetek USA, Inc., Torrance, CA, USA) and attached to microscope slides by heating in an oven for 2 hours at $60^{\circ} \mathrm{C}$. The paraffin sections were rehydrated in three baths of xylenes followed by three baths of graded ethanols. A histological grade of xylene (Fischer Scientific) was used for three baths at $100 \%$ xylene. A histological grade of reagent ethanol (Fischer Scientific) was used for the two baths at $100 \%$ followed by one bath of $95 \%$ ethanol and rinse for 1 minute with tap water. To determine the presence of AuNRs in the tissue, the sections were stained with silver intensification according to the "Silver enhancement procedure" in the Technical Bulletin for the Silver Enhancer Kit (SE100; Sigma Aldrich), followed by staining with hematoxylin and light eosin (Surgipath by Leica Biosystems, Richmond, IL, USA).

\section{Statistical analysis}

Repeated measures on analysis of variance was performed in order to determine whether the combination of nanorods and laser treatment had a different effect on tumor regression than laser-only, nanorod-only, and no-treatment controls. There was significant interaction between the treatment group and specific time points $(P<0.0001)$, suggesting that the changes in tumor volume over time were not the same for all treatment groups. Therefore, one-way analyses of variance were carried out at each time point to examine differences in tumor volume between treatment groups (Table 1). Linear contrasts were generated to examine specifically whether or not average tumor sizes were different between the combination nanorods and laser treatment compared with no-treatment, laser-only, and nanorod-only controls. Additional comparisons were made to identify the differences between the combination nanorod and laser treatment with the averages of each of the other control treatments. Statistical analysis was performed using SAS version 9.1.3 (SAS Institute Inc., Cary, NC, USA), with $P$-values $<0.05$ deemed to be statistically significant.

\section{Results}

The overlap in the NIR spectral range of the laser emission wavelength and the plasmon resonance absorption resulted in rapid electron oscillation within the AuNRs and consequent local heat generation transferred to the surrounding tumor. The combined effect of incubating AuNRs and 4 minutes of NIR light exposure or directly injecting AuNRs into subcutaneous tumors, followed by a single 10 minute treatment with NIR light, caused statistically significant increased cell death or tumor regression respectively.

In vitro cell death resulting from the combination of AuNR incubation and laser treatment, and the control groups (no-treatment, laser-only, and AuNR-only ) are compared in Figure 2A-C. Cells exposed to laser-only treatment, delineated by the oval outline in Figure 2A, appear comparable in viability to cells that were not exposed to laser treatment. Cells exposed to AuNRs only were not dark blue, an indication of cell death that was observed only in cells exposed to both AuNRs and the NIR laser, and delineated by the oval outline in Figure 2B. In comparison, cell death was $<10 \%$ for the no-treatment, laser-only, and AuNR-only control groups, versus $\sim 95 \%$ cell death observed for the combination of AuNRs and laser irradiation (Figure 2C). Cell counts were expressed as the mean value of triplicate observations.

Table I Statistical analysis of tumor regression

\begin{tabular}{|c|c|c|c|c|c|c|c|}
\hline \multirow[t]{2}{*}{ Treatment comparisons } & \multicolumn{7}{|c|}{$P$-values for post-treatment days $I-I 0$} \\
\hline & $\mathbf{I}$ & 2 & 3 & 5 & 7 & 9 & 10 \\
\hline Nanorods + laser vs no treatment & - & 0.0012 & 0.0005 & $<0.0001$ & $<0.0001$ & 0.0001 & $<0.0001$ \\
\hline Nanorods + laser vs laser only & - & 0.0058 & 0.0081 & 0.006 & 0.0056 & 0.0012 & 0.005 \\
\hline Nanorods + laser vs nanorods only & - & 0.0052 & 0.0028 & 0.0037 & 0.1163 & 0.108 & 0.0565 \\
\hline Nanorods + laser vs single treatment avg & - & 0.0016 & 0.0014 & 0.0004 & 0.0102 & 0.0038 & 0.0014 \\
\hline Nanorods + laser vs three control groups' avg* & - & 0.0005 & 0.0003 & $<0.0001$ & 0.0006 & 0.0004 & $<0.0001$ \\
\hline
\end{tabular}

Notes: Observations commenced one day post-treatment (day 2). P-values are stratified by day using ANOVA (one-way analysis of variance) to compare regression between combination treatment (AuNR plus NIR laser irradiation) vs three controls (the no-treatment, laser-only, and nanorod-only groups). The - symbol denotes no data available. *There was a statistically significant difference between the tumor sizes of the combination nanorod and laser treatment group ( $\mathrm{n}=6$ ) and the average of all other groups $(n=I I)$ across all time points $(P<0.0 I)$

Abbreviations: avg, average; vs, versus; NIR, near infrared; AuNR, gold nanorod. 

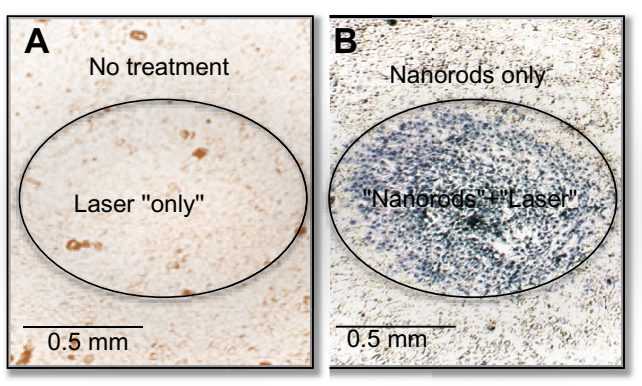

\section{C}

D

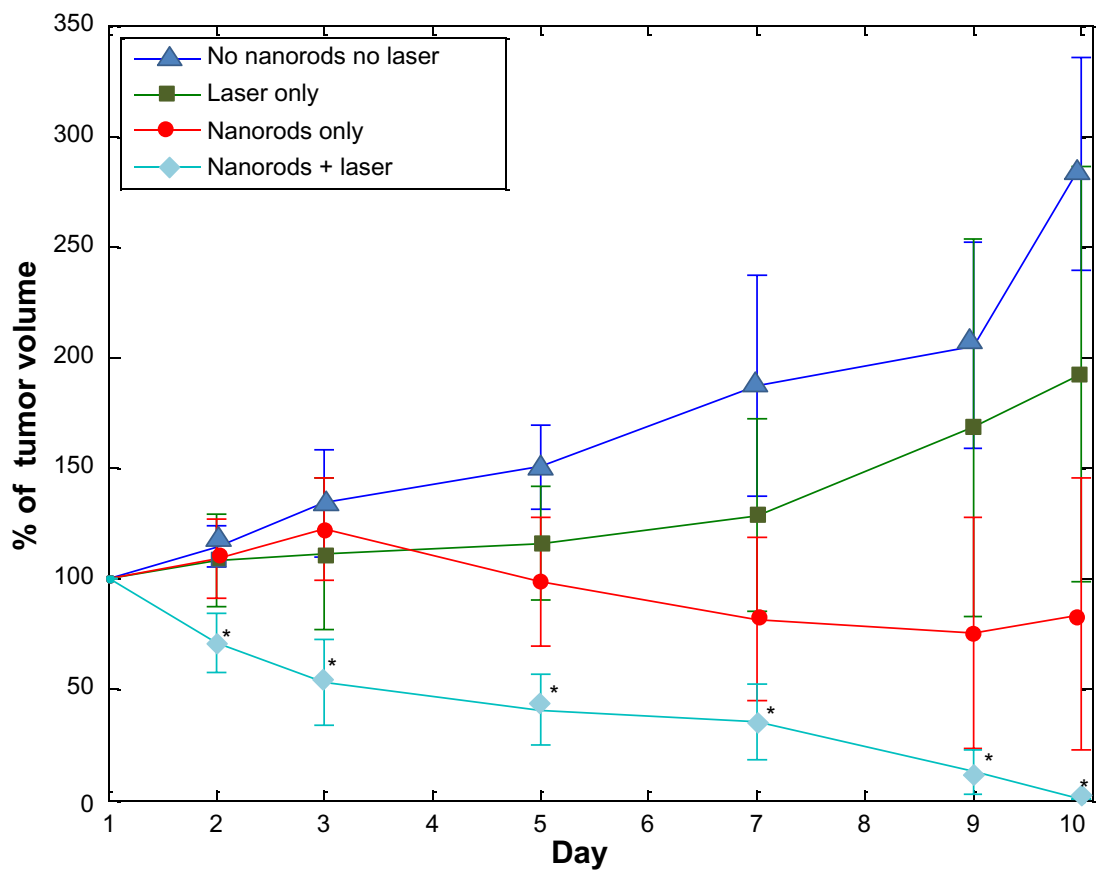

Figure 2 An in vitro (A-C) and in vivo (D) summary comparing the nanorod and laser combination treatment to the three controls: the no-treatment, laser-only, and nanorod-only groups.

Notes: A cell viability assay using trypan blue staining (which causes dead cells to turn dark blue) for cells treated (A) with no nanorods, with (inside oval) and without (outside oval) laser irradiation and (B) with nanorods, with (inside oval) and without (outside oval) laser irradiation. The oval shape indicates laser contact area with the tumor. (C) An in vitro summary of the percentages of cell death due to each treatment. (D) An in vivo summary of the average changes in tumor volume over I0 days. Cell counts expressed as the mean value of triplicate observations. *Indicates a statistically significant difference between the combination treatment and the average of the other three control groups. The error bars are resolved from standard deviation calculations ( \pm standard deviation).

Tumor volumes after exposure to the combination of AuNRs and NIR laser $(n=6)$, and tumor volumes of the three control groups, which were the no-treatment $(\mathrm{n}=4)$, laser-only $(n=4)$, and AuNR-only ( $n=3)$, groups, were compared. The measured changes in tumor volumes over 10 days for each of the different treatment groups, summarized in Figure 2D, were analyzed for statistical significance on each day (Table 1), and were subjected to histological examination on day 10 (Figure 3). A decrease in tumor volume was observed (on day 1) after the combination treatment of intratumoral AuNR injections and a single 10-minute laser irradiation (nanorod and laser), as compared to the control groups starting on the day after treatment (day 2), and this decrease was maintained for the duration of the study. Tumor volumes in the no-treatment control group increased to nearly three times the original tumor volume. Tumors in the laser-only control group, which sustained NIR laser irradiation for 10 minutes at $785 \mathrm{~nm}$, and $9.5 \mathrm{~W} / \mathrm{cm}^{2}$, grew to approximately double the original volume, displaying slightly modified growth patterns. Tumors directly injected with PEGylated AuNRs without any laser exposure (the nanorod-only control group) showed inhibited growth patterns, not regression, and roughly maintained original tumor volume. There was a statistically significant difference between the tumor sizes of the combination nanorod and laser treatment group ( $\mathrm{n}=6)$ and the average of all other groups $(\mathrm{n}=11)$ across all time points $(P<0.01)$.

Histological examination of representative excised tumors provided insight into the consequences of each of the treatments (Figure 3). These results are quantified and compared in Figure 2. Tumor histology was evaluated by a 


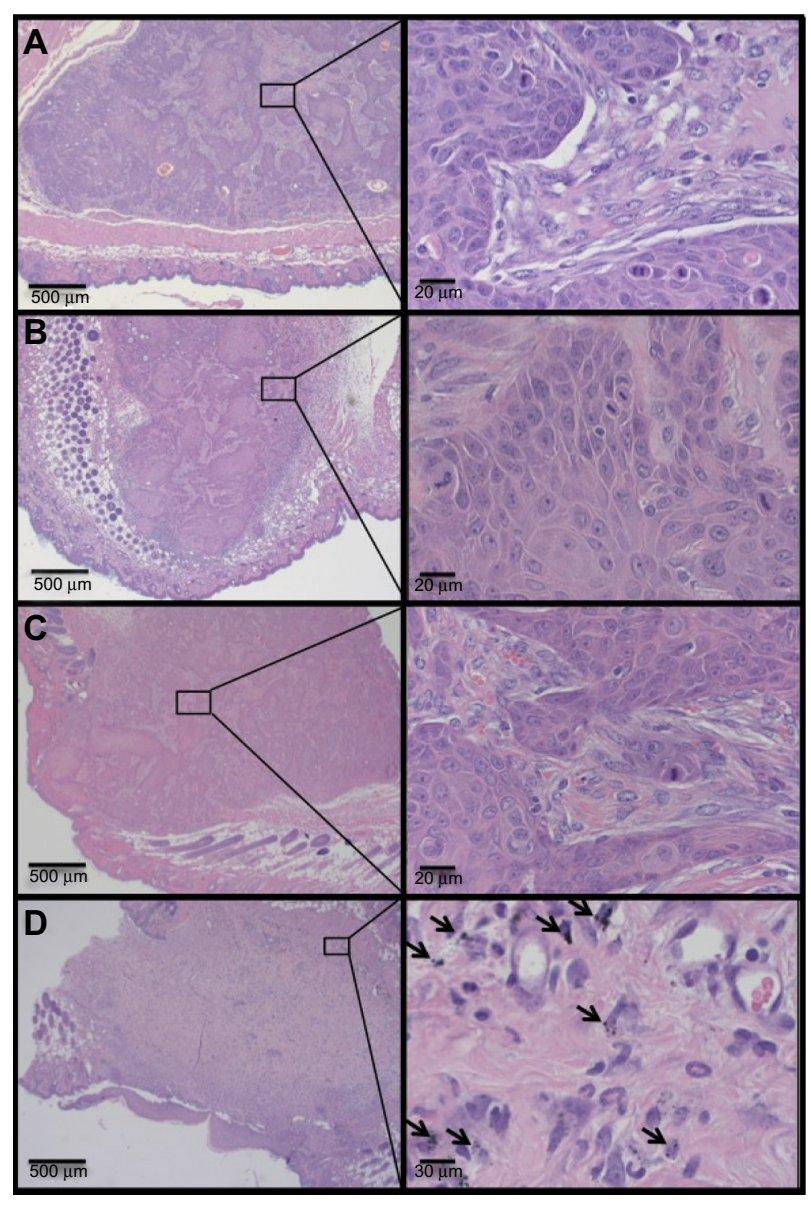

Figure 3 Histology of representative tumors excised after 10 days of treatment. Notes: Comparing all four treatment groups: (A) no treatment presenting viable tumor, (B) laser-only treatment showing viable tumor, (C) nanorod-only treated tissue consisting of mostly viable tumor, and (D) combination nanorod and laser treatment with no definitely identifiable tumor, only reparative changes including fibrosis and inflammatory cells. Silver intensification demonstrated gold particles were present in the areas of damage (black arrows). Images on the right are insets of images on the left.

pathologist (William E Grizzle) certified by the American board of Pathology (Tampa, FL, USA). Excised tumor tissue from the no treatment control group (Figure $3 \mathrm{~A}$ ) was used as the standard to identify viable tumor structure versus other tissues present throughout the treatment groups. Untreated tumor characteristics include distinct cellular boundaries indicating intact cells; the presence of mitosis indicating tumor proliferation; and some intrinsic tumor death and tissue damage likely indicating ischemia, generally caused by inadequate vasculature supply (Figure 3A, inset). Characteristics of tumor tissue treated with only the NIR laser (Figure 3B) are consistent with those of no-treatment tumor tissue (Figure 3A), showing mitoses and intact cells (Figure 3B, inset). In nanorod-only treated tumors, there was AuNR accumulation in areas near the disturbed blood vessels, coinciding with nanorod injection sites (Figure 3C).
This association of tumor damage with vascular disruption is contrasted with histological examinations showing undisturbed viable tumor distant from vascular disruption near injection sites (Figure 3C, inset); this association was also found in the no-treatment and laser-only treatment groups (Figure 3A and B). The areas under and around the scabs or scars remaining after the combination treatment of nanorods and laser, as opposed to the tumors shown in Figure 3A-C, were examined microscopically (Figure 3D). Damage was apparent to the surface epithelial and hair follicles. Fibrosis and inflammatory cells were observed, suggesting tissue repair. The histological pattern of combination nanorod and laser post-treatment tissue (Figure 3D, inset) was very different from the cohesive cells of viable tumor tissue noted in Figure $3 \mathrm{~A}-\mathrm{C}$, and nanorods were visible in the area of damage. No definitive residual tumor tissue was identified.

Acute damage to tumor tissue and dramatic reduction in tumor volume was observed after the combination nanorod and laser treatment only, not after any of the other treatment groups. This rapid tumor regression was captured in the photographic images taken on day 1, before and after treatment, then daily until the 15th day after treatment (Figure 4A-N). The regressed tumor experienced immediate discoloration after treatment. Tumor regression continued for 15 days after the combination nanorod and laser treatment, and these gross changes in morphology were representative of all tumors treated with the combination therapy.

Post-treatment tumor histology on day 2 (Figure 5A), on day 7 (Figure 5B), and on day 15 (Figure 5C) correlate by day with photographic images shown in Figure $4 \mathrm{C}$, $\mathrm{H}$, and $\mathrm{N}$, respectively. On day 2 , the first day after the combination treatment, tumor damage included coagulation and disruption to the skin and hair follicles. Blood vessel damage and the presence of red blood cells in the tumor indicate the level of cellular destruction (Figure 5A). Tumor histology at 7 and 15 days after the combination nanorod and laser treatment (Figure 5B and C) shows no indication of viable tumor; only inflammatory cells, fibrosis, and intact but edematous skin remain. The presence of macrophages with brown pigment, which contain AuNPs and the degradation products of red blood cells (hemosiderin), are consistent with the removal of necrotic debris and active cellular repair.

In summary, $\sim 100 \%$ tumor regression was attained in $\sim 90 \%$ ( 14 of 16) of combination treated tumors. No definitive, viable tumor was identified by histological examination on day 15 after treatment. 


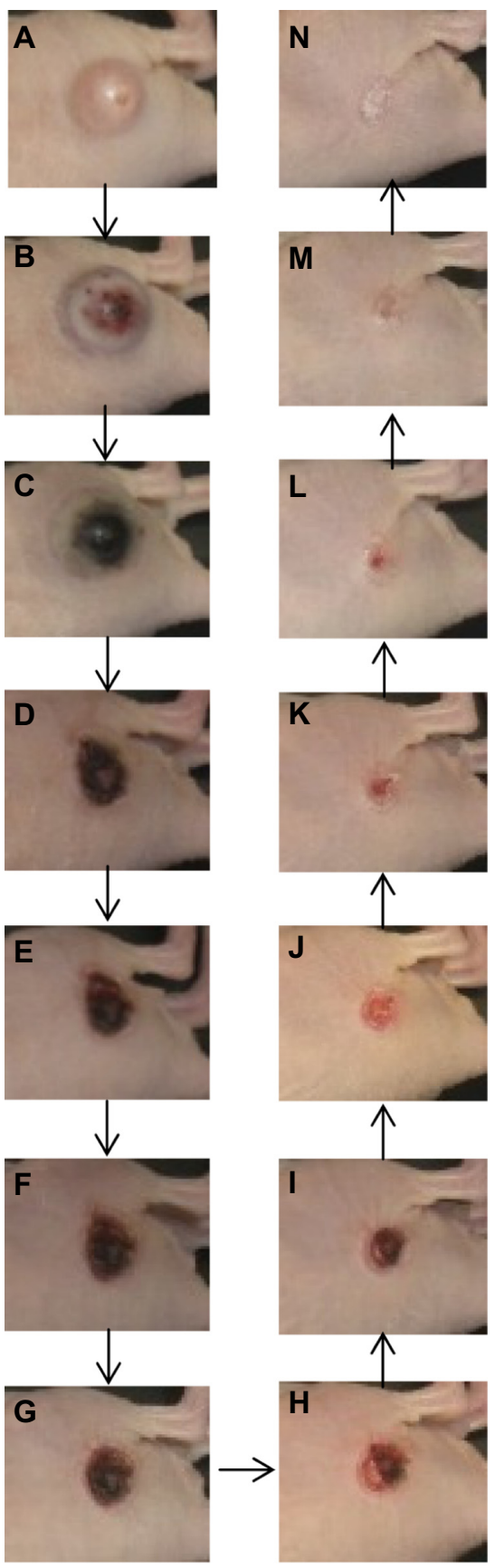

Figure 4 Photographic monitoring of tumor regression over 15 days in response to photothermal therapy, intratumoral injections of PEGylated AuNRs and a single 10-minute NIR laser irradiation.

Notes: (A) Day I before treatment; (B) day I after treatment; (C) day 2, (D) day 3, (E) day 4, (F) day 5, (G) day 6, (H) day 7, (I) day 8, (J) day 9, (K) day 10, (L) day II, (M) day 13, and (N) day 15. The width of all cropped pictures is $19.05 \mathrm{~mm}$ (0.75 in).

Abbreviations: PEG, polyethylene glycol; in, inches; AuNRs, gold nanorods.

\section{Discussion}

The combined effect of directly injecting AuNRs into subcutaneous tumors followed by a single 10 minute treatment with NIR light caused statistically significant tumor regression over 10 days, and in most cases, an apparent elimina-

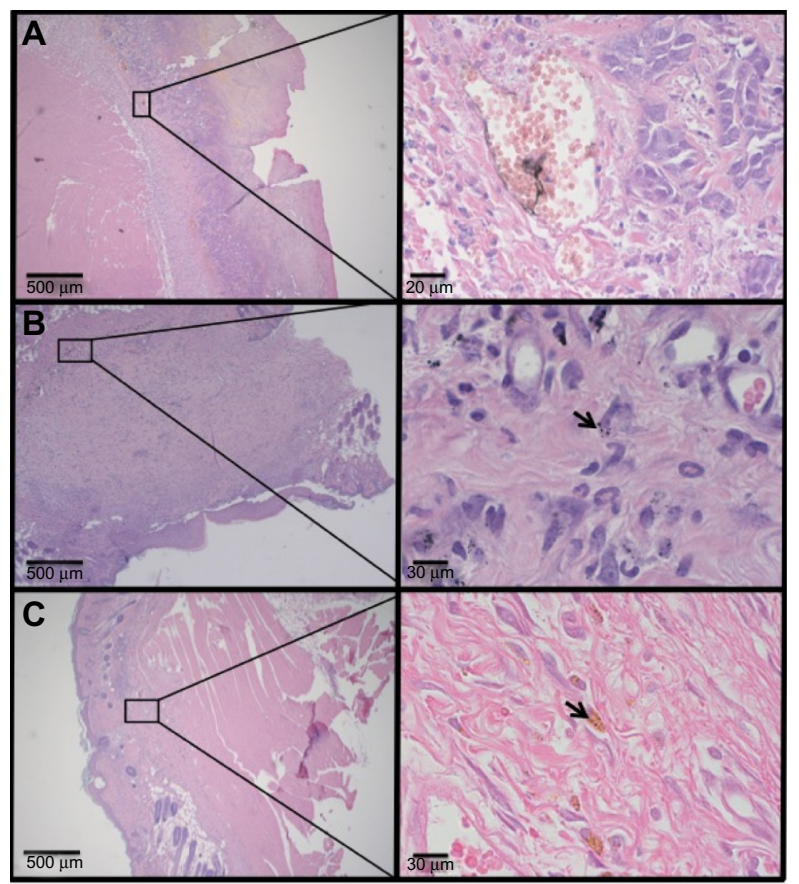

Figure $\mathbf{5}$ Histology of tumors excised at different time points after photothermal nanotherapy, treatment with directly injected PEGylated AuNRs and laser irradiation.

Notes: (A) Day 2, (B) day 7, and (C) day 15. Black arrows indicate nanorods before and after uptake by a macrophage containing a pigment, probably hemosiderin stain. Images on the right (magnification 25x) are insets of images on the left (magnification 400x). Abbreviations: PEG, polyethylene glycol; AuNRs, gold nanorods.

tion of the tumor. The changes in tumor volume for each of the different treatment and control groups were analyzed for statistical significance on each day, and tumor tissue was histologically examined on day 10 . A similar trend in tumor regression was reported for PEGylated AuNRs ${ }^{42}$ and chitosan-conjugated AuNR nanocarriers, ${ }^{43}$ but only after repeated laser treatments. These studies differ from ours in that both non-targeted and tumor-targeted NP delivery mechanisms enabled tumor site delivery of $<20 \%$ of injected NPs; $\sim 80 \%-90 \%$ of the injected NPs were accumulated in the spleen, liver, kidney, lung, and heart. These reports also contained multiple treatments and lacked both statistical and histological evaluations.

NIR light is reported not to cause cellular death; ${ }^{43}$ this is consistent with our analysis that the observed differences between the changes in tumor volumes of the laser-only and the no-treatment control groups was not statistically significant. The nanorod-only treatment group did not display the growth patterns observed in the other control groups. Our in vitro data is consistent with previous studies demonstrating the non-cytotoxic behavior of PEGylated AuNPs. ${ }^{19,37,38}$ The non-cytotoxic behavior of PEGylated AuNRs implies that they are biologically inert and do not intrinsically and 
independently cause cell death without acting in combination with light exposure. Therefore, the inhibited growth pattern observed in the nanorod-only treatment group in our vivo studies is not likely due to any cytotoxicity of the nanorods. ${ }^{54}$ It is possible, however, that observed growth inhibition was due to prolonged exposure to ambient light. Elongated NPs like nanorods have two surface plasmon resonance absorption peaks, the longitudinal and transverse modes, corresponding to NIR itself and surrounding, ambient visible light, respectively. ${ }^{27}$ Thus, it is likely that ambient light absorption along the transverse mode of the nanorods contributed to a photothermal effect on the tumor, which modified growth patterns. It is also possible that observed modified growth patterns of endothelial cells could be attributed to superficial blood vessel injury, which was more likely due to the multiple injections, not the nanorods themselves. Future studies that include larger sample sizes, ambient light controls, and multiple phosphate buffered saline-only injections would clarify this issue, and address the limitations of this study.

Histological analysis of the combination-treated tumor, after direct injection with AuNRs followed by a single 10 minute NIR laser treatment, showed that externally located black discoloration of treated tumors was probably due to blood leaking from physically as well as thermally disrupted vessels. It is likely that the destruction of blood vessels (leading to cellular death), in addition to the immediate temperature-induced damage (leading to necrosis and coagulation), caused the continued reduction in tumor volume that was observed over the duration of the study.

It is known that tumor recurrence is reduced when the tumor is treated inclusive of its junction with normal tissue (ie, the tumor margin). Similar to other treatments, PNT is most effective if the entire tumor and its interface with healthy tissue are treated. Even though the evaluation of tumor recurrence is beyond the scope of this study, our findings suggest that PNT is capable of targeting multiple cell types contained within a tumor, which can reduce the likelihood of recurrence. Uniform distribution of nanorods throughout the tumor and an appropriate diameter laser are both essential to obtain uniform results.

\section{Conclusion}

The combination treatment of AuNRs and NIR laser demonstrates $\sim 100 \%$ efficiency in tumor regression within 10-15 days in $\sim 90 \%$ of the tumors treated. Direct intratumoral injections as a novel approach for the delivery of AuNRs effectively overcomes the low efficiency observed in other in vivo NP delivery systems in which the RES uptake was only $80 \%-90 \%$ of the injected NPs. Even though this study used a specific cancer type, oral squamous carcinoma, and subcutaneous xenograft mouse models, intratumoral injection of AuNRs and subsequent photothermal treatment of tumors is a promising approach that may translate into an effective treatment for a variety of cancer types in humans. Head and neck, colorectal, ovarian, skin, cervical, breast, bladder, pancreatic, and prostate cancers are ideal candidates for trial because they are readily accessible by lasers and are all within the $10 \mathrm{~cm}$ penetration depth of NIR light. Furthermore, this novel approach may be a realistic option for treating other solid and early stage tumors in combination with early detection and imaging mechanisms; however, whether PNT is appropriate for the treatment of metastatic tumors is yet to be determined. Future studies should address the morphological and genetic analysis of residual tissue and the impact of ambient light on tumors. Furthermore, development of additional clinical applications, such as PNT combined with the current standard of care, is a rational approach for realizing new therapies.

\section{Acknowledgments}

This work has been funded by the US Department of Education Graduate Assistance in Areas of Need (GAANN) Fellowship; the National Physical Science (NSF) Consortium Fellowship, (grant EPS-0814103), the US National Institutes of Health/National Institutes of Dental and Craniofacial Research developmental research (NIH/ NIDCR R21) grant (number 1R21DE019232-01A2); the U54 Morehouse School of Medicine/Tuskegee University/ University of Alabama (UAB) Comprehensive Cancer Center Partnership (grant 2U54CA118948); and the National Institutes of Health (NIH) (grant number P30 CA13148). We acknowledge the UAB TEM Facility, Clayton Yates, Paul Castellanos, Denise Oelschlager, Rob Stockard, Lacey McNally, Angela Brown, Adolphus Elliot, and Brian Sims for encouragement, technical instruction, and supportive discussions.

\section{Disclosure}

The authors report no conflicts of interest in this work.

\section{References}

1. Saldanha DF, Khiatani VL, Carrillo TC, et al. Current tumor ablation technologies: basic science and device review. Semin Intervent Radiol. 2010;27(3):247-254.

2. Zhao Z, Wu F. Minimally-invasive thermal ablation of early-stage breast cancer: a systemic review. Eur J Surg Oncol. 2010;36(12): 1149-1155.

3. Iberti CT, Mohamed N, Palese MA. A review of focal therapy techniques in prostate cancer: clinical results for high-intensity focused ultrasound and focal cryoablation. Rev Urol. 2011;13(4):e196-e202. 
4. Hall-Craggs MA, Vaidya JS. Minimally invasive therapy for the treatment of breast tumours. Eur J Radiol. 2002;42(1):52-57.

5. Martin RC, Scoggins CR, McMasters KM. Safety and efficacy of microwave ablation of hepatic tumors: a prospective review of a 5-year experience. Ann Surg Oncol. 2010;17(1):171-178.

6. Wasser EJ, Dupuy DE. Microwave ablation in the treatment of primary lung tumors. Semin Respir Crit Care Med. 2008;29(4):384-394.

7. Stańczyk M, Zegadlo A, Zwierowicz T, Zak D, Boguslawska R, Maruszyński M. [Microwave ablation of liver tumors as a new instrument for minimally invasive liver surgery]. Pol Merkur Lekarski. 2009;26(155):545-549. Polish [English abstract].

8. Gorny KR, Woodrum DA, Brown DL, et al. Magnetic resonance-guided focused ultrasound of uterine leiomyomas: review of a 12-month outcome of 130 clinical patients. J Vasc Interv Radiol. 2011;22(6): 857-864.

9. Kopelman D, Papa M. Magnetic resonance-guided focused ultrasound surgery for the noninvasive curative ablation of tumors and palliative treatments: a review. Ann Surg Oncol. 2007;14(5):1540-1550.

10. Zippel DB, Papa MZ. The use of MR imaging guided focused ultrasound in breast cancer patients; a preliminary phase one study and review. Breast Cancer. 2005;12(1):32-38.

11. Bojalian MO, Machado GR, Swensen R, Reeves ME. Radiofrequency ablation of liver metastasis from ovarian adenocarcinoma: case report and literature review. Gynecol Oncol. 2004;93(2):557-560.

12. Haïssaguerre M, Gaïta F, Marcus FI, Clementy J. Radiofrequency catheter ablation of accessory pathways: a contemporary review. J Cardiovasc Electrophysiol. 1994;5(6):532-552.

13. Maccini M, Sehrt D, Pompeo A, Chicoli FA, Molina WR, Kim FJ Biophysiologic considerations in cryoablation: a practical mechanistic molecular review. Int Braz J Urol. 2011;37(6):693-696.

14. Mala T. Cryoablation of liver tumours - a review of mechanisms, techniques and clinical outcome. Minim Invasive Ther Allied Technol. 2006;15(1):9-17.

15. Zippe $\mathrm{CD}$. Cryosurgical ablation for prostate cancer: a current review. Semin Urol. 1995;13(2):148-156.

16. Cai W, Gao T, Hong H, Sun J. Applications of gold nanoparticles in cancer nanotechnology. Nanotechnol Sci Appl. 2008;(1):17-32.

17. Choi J, Yang J, Jang E, et al. Gold nanostructures as photothermal therapy agent for cancer. Anticancer Agents Med Chem. 2011;11(10): 953-964.

18. Huang X, Jain PK, El-Sayed IH, El-Sayed MA. Plasmonic photothermal therapy (PPTT) using gold nanoparticles. Lasers Med Sci. 2008;23(3):217-228.

19. Loo C, Lowery A, Halas N, West J, Drezek R. Immunotargeted nanoshells for integrated cancer imaging and therapy. Nano Lett. 2005;5(4):709-711.

20. Morton JG, Day ES, Halas NJ, West JL. Nanoshells for photothermal cancer therapy. In: Grobmyer SR, Moudgil BM, editors. Cancer Nanotechnology: Methods and Protocols, Methods in Molecular Biology Series 624. New York: Humana Press; 2010:101-117.

21. Gobin AM, Lee MH, Halas NJ, James WD, Drezek RA, West JL. Near-infrared resonant nanoshells for combined optical imaging and photothermal cancer therapy. Nano Lett. 2007;7(7):1929-1934.

22. Skrabalak SE, Au L, Lu X, Li X, Xia Y. Gold nanocages for cancer detection and treatment. Nanomedicine (Lond). 2007; 2(5):657-668.

23. Skrabalak SE, Chen J, Au L, Lu X, Li X, Xia Y. Gold Nanocages for Biomedical Applications. Adv Mater. 2007;19(20):3177-3184.

24. Huang X, El-Sayed IH, El-Sayed MA. Applications of gold nanorods for cancer imaging and photothermal therapy. Methods Mol Biol. 2010;624:343-357.

25. Dickerson EB, Dreaden EC, Huang X, et al. Gold nanorod assisted near-infrared plasmonic photothermal therapy (PPTT) of squamous cell carcinoma in mice. Cancer Lett. 2008;269(1):57-66.

26. Goodrich GP, Bao L, Gill-Sharp K, Sang KL, Wang J, Payne JD. Photothermal therapy in a murine colon cancer model using nearinfrared absorbing gold nanorods. J Biomed Opt. 2010;15(1):018001.
27. Jain PK, Lee KS, El-Sayed IH, El-Sayed MA. Calculated absorption and scattering properties of gold nanoparticles of different size, shape, and composition: applications in biological imaging and biomedicine. J Phys Chem B. 2006;110(14):7238-7248.

28. Kennedy LC, Bickford LR, Lewinski NA, et al. A new era for cancer treatment: gold-nanoparticle-mediated thermal therapies. Small. 2011;7(2):169-183.

29. Yang Y, Wang H. Perspectives of nanotechnology in minimally invasive therapy of breast cancer. J Healthc Eng. 2013;4(1):67-86.

30. Yeh YC, Creran B, Rotello VM. Gold nanoparticles: preparation, properties, and applications in bionanotechnology. Nanoscale. 2012; 4(6): $1871-1880$

31. Zhang Z, Wang J, Chen C. Gold nanorods based platforms for lightmediated theranostics. Theranostics. 2013;3(3):223-238.

32. Zhao J, Pinchuk AO, McMahon JM, et al. Methods for describing the electromagnetic properties of silver and gold nanoparticles. Acc Chem Res. 2008;41(12):1710-1720.

33. Huang X, Jain PK, El-Sayed IH, El-Sayed MA. Gold nanoparticles: interesting optical properties and recent applications in cancer diagnostics and therapy. Nanomedicine (Lond). 2007;2(5):681-693.

34. Choi YJ, Kim YJ, Lee JW, et al. Cytotoxicity and genotoxicity induced by photothermal effects of colloidal gold nanorods. J Nanosci Nanotechnol. 2013;13(6):4437-4445.

35. Rejiya CS, Kumar J, Raji V, Vibin M, Abraham A. Laser immunotherapy with gold nanorods causes selective killing of tumour cells. Pharmacol Res. 2012;65(2):261-269.

36. Manthe RL, Foy SP, Krishnamurthy N, Sharma B, Labhasetwar V. Tumor ablation and nanotechnology. Mol Pharm. 2010;7(6):1880-1898.

37. Huang X, El-Sayed IH, Qian W, El-Sayed MA. Cancer cell imaging and photothermal therapy in the near-infrared region by using gold nanorods. J Am Chem Soc. 2006;128(6):2115-2120.

38. El-Sayed IH, Huang X, El-Sayed MA. Selective laser photo-thermal therapy of epithelial carcinoma using anti-EGFR antibody conjugated gold nanoparticles. Cancer Lett. 2006;239(1):129-135.

39. Maeda $H$. The enhanced permeability and retention (EPR) effect in tumor vasculature: the key role of tumor-selective macromolecular drug targeting. Adv Enzyme Regul. 2001;41:189-207.

40. Greish K. Enhanced permeability and retention (EPR) effect for anticancer nanomedicine drug targeting. Methods Mol Biol. 2010;624: 25-37.

41. Fang J, Nakamura H, Maeda H. The EPR effect: Unique features of tumor blood vessels for drug delivery, factors involved, and limitations and augmentation of the effect. Adv Drug Deliv Rev. 2011;63(3): 136-151.

42. Li Z, Huang P, Zhang X, et al. RGD-conjugated dendrimer-modified gold nanorods for in vivo tumor targeting and photothermal therapy. Mol Pharm. 2010;7(1):94-104.

43. Choi WI, Kim JY, Kang C, Byeon CC, Kim YH, Tae G. Tumor regression in vivo by photothermal therapy based on gold-nanorod-loaded, functional nanocarriers. ACS Nano. 2011;5(3):1995-2003.

44. Abdelhalim MA. Uptake of gold nanoparticles in several rat organs after intraperitoneal administration in vivo: a fluorescence study. Biomed Res Int. 2013:353695.

45. De Jong WH, Hagens WI, Krystek P, Burger MC, Sips AJ, Geertsma RE. Particle size-dependent organ distribution of gold nanoparticles after intravenous administration. Biomaterials. 2008;29(12):1912-1919.

46. Chen H, Dorrigan A, Saad S, Hare DJ, Cortie MB, Valenzuela SM. In vivo study of spherical gold nanoparticles: inflammatory effects and distribution in mice. PLoS One. 2013;8(2):e58208.

47. Abdelhalim MA, Abdelmottaleb Moussa SA. The gold nanoparticle size and exposure duration effect on the liver and kidney function of rats: In vivo. Saudi J Biol Sci. 2013;20(2):177-181.

48. Lankveld DP, Rayavarapu RG, Krystek P, et al. Blood clearance and tissue distribution of PEGylated and non-PEGylated gold nanorods after intravenous administration in rats. Nanomedicine (Lond). 2011;6(2):339-349.

49. Sonavane G, Tomoda K, Makino K. Biodistribution of colloidal gold nanoparticles after intravenous administration: effect of particle size. Colloids Surf B Biointerfaces. 2008;66(2):274-280. 
50. Sau TK, Murphy CJ. Seeded high yield synthesis of short Au nanorods in aqueous solution. Langmuir. 2004;20(15):6414-6420.

51. Green HN, Martyshkin DV, Rodenburg CM, Rosenthal EL, Mirov SB. Gold nanorod bioconjugates for active tumor targeting and photothermal therapy. J Nanotechnol. 2011;2011:1-7.

52. Shan L. Polyethylene glycol-coated (PEG5000) gold nanoparticles. Molecular Imaging and Contrast Agent (MICAD) Database [database on the Internet]. Bethesda, MD: National Center for Biotechnology Information. Available from: http://www.ncbi.nlm.nih.gov/pubmed/. Accessed June 1, 2012.
53. Niidome T, Yamagata M, Okamoto Y, et al. PEG-modified gold nanorods with a stealth character for in vivo applications. J Control Release. 2006;114(3):343-347.

54. Alkilany AM, Shatanawi A, Kurtz T, Caldwell RB, Caldwell RW. Toxicity and cellular uptake of gold nanorods in vascular endothelium and smooth muscles of isolated rat blood vessel: importance of surface modification. Small. 2012;8(8):1270-1278.

\section{Publish your work in this journal}

The International Journal of Nanomedicine is an international, peerreviewed journal focusing on the application of nanotechnology in diagnostics, therapeutics, and drug delivery systems throughout the biomedical field. This journal is indexed on PubMed Central, MedLine, CAS, SciSearch ${ }^{\circledR}$, Current Contents ${ }^{\circledR} /$ Clinical Medicine,
Journal Citation Reports/Science Edition, EMBase, Scopus and the Elsevier Bibliographic databases. The manuscript management system is completely online and includes a very quick and fair peer-review system, which is all easy to use. Visit http://www.dovepress.com/ testimonials.php to read real quotes from published authors. 